\title{
Small Interference RNA Targeting TLR4 Gene Effectively Attenuates Pulmonary Inflammation in a Rat Model
}

\author{
Feixiang Wu, Yantao Liu, Xin Lv, Xuerong Miao, Yuming Sun, and Weifeng Yu \\ Department of Anesthesiology, Eastern Hepatobiliary Hospital, No. 225 Road Changhai, Shanghai 200433, China \\ Correspondence should be addressed to Weifeng Yu, ywf808@sohu.com
}

Received 12 July 2011; Revised 17 October 2011; Accepted 28 October 2011

Academic Editor: Masamitsu Yamaguchi

Copyright () 2012 Feixiang Wu et al. This is an open access article distributed under the Creative Commons Attribution License, which permits unrestricted use, distribution, and reproduction in any medium, provided the original work is properly cited.

\begin{abstract}
Objective. The present study was to investigate the feasibility of adenovirus-mediated small interference RNA (siRNA) targeting Toll-like receptor 4 (TLR4) gene in ameliorating lipopolysaccharide- (LPS-) induced acute lung injury (ALI). Methods. In vitro, alveolar macrophages (AMs) were treated with Ad-siTLR4 and Ad-EFGP, respectively, for $12 \mathrm{~h}, 24 \mathrm{~h}$, and $48 \mathrm{~h}$, and then with LPS $(100 \mathrm{ng} / \mathrm{mL})$ for $2 \mathrm{~h}$, and the function and expression of TLR4 were evaluated. In vivo, rats received intratracheal injection of $300 \mu \mathrm{L}$ of normal saline (control group), $300 \mu \mathrm{L}$ of Ad-EGFP (Ad-EGFP group), or $300 \mu \mathrm{L}$ of Ad-siTLR4 (Ad-siTLR4 group) and then were intravenously treated with LPS $(50 \mathrm{mg} / \mathrm{kg}$ ) to induce ALI. Results. Ad-siTLR4 treatment significantly reduced TLR4 expression and production of proinflammatory cytokines following LPS treatment both in vitro and in vivo. Significant alleviation of tissue edema, microvascular protein leakage, and neutrophil infiltration was observed in the AdsiTLR4-treated animals. Conclusion. TLR4 plays a critical role in LPS-induced ALI, and transfection of Ad-siTLR4 can effectively downregulate TLR4 expression in vitro and in vivo, accompanied by alleviation of LPS-induced lung injury. These findings suggest that TLR4 may serve as a potential target in the treatment of ALI and RNA interfering targeting TLR4 expression represents a therapeutic strategy.
\end{abstract}

\section{Introduction}

Pulmonary trauma, severe infection as well as hemorrhage can result in acute lung injury (ALI) or acute respiratory distress syndrome (ARDS), which are common pulmonary diseases in humans, especially in patients undergoing surgery [1-4]. Studies have confirmed that lipopolysaccharide (LPS) plays important roles in the development and progression of ALI or ARDS [5]. Increased serum endotoxin or LPS of Gram-negative bacteria may induce pulmonary inflammation leading to ALI and can be applied as a predictor of multiple organ failure (MOF), particularly during sepsis. LPS recognition by the host receptor is a critical step leading to the activation of signal transduction cascades in a variety of cells in the lung [6-8]. It is widely believed that Toll-like receptor 4 (TLR4) is required for the innate immune response to LPS of gram-negative bacteria $[9,10]$. Studies have shown that mice with TLR4 gene deletion $\left(\mathrm{TLR}^{-/-}\right)(\mathrm{C} 57 \mathrm{BL} / 10 \mathrm{ScCr})[9]$ and mice with natural point mutation $(\mathrm{C} 3 \mathrm{H} / \mathrm{HeJ})$ in TLR4 gene [10] are unresponsive to systemic LPS. However, the role of TLR4 in the pulmonary inflammation is still debatable. Some researchers speculate that it is LPS other than TLR4 that is directly involved in the induction of pulmonary immune response [11]. However, Jeyaseelan et al. demonstrated that LPS-induced CD-14-dependent and -independent (CD11b-dependent) signaling pathways in the lung were entirely dependent on TLR4 and that blocking TLR4 might be beneficial for lung injury caused by LPS from gram-negative pathogens [12].

With the progression in the technology of gene therapy, RNA interference (RNAi) mediated by small interfering RNAs (siRNA) has emerged as a powerful tool to knock down target gene in cells and as a gold standard in the studies on gene function [13]. Evidence has shown that the 21 and 22 nucleotide RNAs are able to mediate the cleavage of target RNA [14-16]. These RNAs are termed as siRNA, and increasing attention has been paid to the development of new target-specific drugs. Studies have revealed that siRNAs are involved in the degradation of not only homologous RNAs in mammalian cells, but also specific target sequence of hepatitis $\mathrm{C}$ virus in living mice and 
vascular endothelial growth factor (VEGF) gene in murine laser-induced choroidal neovascularization (CNV) model [17].

Since LPS recognition by TLR4 is the first step in the pulmonary innate immune response [18, 19], downregulating TLR4 expression or blocking its functions with antibodies may serve as effective therapeutic strategies [15, 16]. Up to now, studies on TLR4-specific blocker are seldom reported and siRNA technique aiming to interfere with TLR4 expression in response to pathogen associated molecules has been one of the promising strategies. In the present study, we designed recombinant adenovirus expressing siRNA targeting TLR4 gene or enhanced green fluorescent protein (EGFP) gene, which was then transfected into macrophages in vitro and rats in vivo. Our study aimed to identify the role of TLR4 in the LPS-induced ALI and evaluate the therapeutic efficacy of TLR4 siRNA in ALI. We for the first time used siRNA targeting TLR4 (Ad-siTLR4) to downregulate TLR4 expression in the lung for the treatment of ALI. Our results demonstrated that transfection with Ad-siTLR4 may be a useful approach for the treatment of TLR4-mediated inflammatory response.

\section{Materials and Methods}

2.1. Construction and Identification of pShuttleH1-siTLR4. The siRNA targeting TLR4 gene (GenBank accession NM_019178) was screened and tested in our previous study [20]. Based on the sequences of adenovirus and "Tuschl" principle, target sequence was designed and chemically synthesized by United Gene Company (Shanghai, China). This siRNA consisted of an RNA duplex containing a sense strand: 5'-GAT CCC CGT CTC AGA TAT CTA GAT CTT TCA AGA GAA GAT CTA GAT ATC TGA GAC TTT TT-3' and an antisense strand: 5'-AGC TAA AAA GTC TCA GAT ATC TAG ATC TTC TCT TGA AAG ATC TAG ATA TCT GAG ACG GG-3'. There were BamH I and Hind III sites at the $5^{\prime}$ and $3^{\prime}$ ends, respectively. After pShuttleH1 was digested by $B g l \mathrm{II}$ and Hind III (TaKaRa, Japan), target gene was cloned into pShuttleH1 and named as pShuttleH1-siRNA. Then, pShuttleH1-siRNA was linearized by Pme I digestion and transformed into E. coli BJ5183 cells containing backbone plasmid pAdEasy- 1 by electroporation. The correct clone was identified by Hind III and Nde I restriction digestion and sequenced by United Gene Company (Shanghai, China).

\subsection{Production and Identification of Recombinant Adenovirus} Ad-siTLR4. To produce recombinant adenovirus Ad-siTLR4 (recombinant adenovirus expressing small interference RNA of TLR4), linearized recombinant plasmid was transfected into HEK 293 cells with Lipofectamine 2000. Plaques were found at about 9 14 days after transfection. To test whether wide-type (WT) virus emerged during the amplification, RTPCR was carried out to detect the E1 region of adenovirus. Plaque purification and titer determination were performed in HEK 293 cells in six-well plates. The titers of adenovirus were measured using the $50 \%$ tissue culture infection dosage (TCID50) method.
2.3. Ad-siTLR4 Transfection and LPS Treatment of Pulmonary Alveolar Macrophages. Isolation and culture of rat alveolar macrophages (AMs) were carried out as previously described [21]. The AMs were applied for viral infection at the multiplicity of infection (MOI) of 20 at 2 days after isolation. AMs were treated with Ad-siTLR4 and Ad-EFGP (recombinant adenovirus expressing enhanced green fluorescent protein (EGFP)), respectively, for $12 \mathrm{~h}, 24 \mathrm{~h}$, and $48 \mathrm{~h}$, and then with LPS $(100 \mathrm{ng} / \mathrm{mL})$ for $2 \mathrm{~h}$. Cells and supernatant were collected for western blot assay, real-time PCR assay, as well as ELISA.

2.4. In Vivo Experiment. The current study was approved by the Animal Care Committee of Second Military Medical University and performed in accordance with National Institutes of Health guidelines for animal use. Male Sprague-Dawley (SD) rats weighing 200-250 g (6-7-week old) were purchased from the Shanghai Experimental Animal Center of Chinese Academy of Sciences and housed in an SPF laboratory under a 12/12 h light/dark cycle. The establishment of ALI model by LPS has been described previously [22]. After anesthesia with pentobarbital sodium $(60 \mathrm{mg} / \mathrm{kg}$, i.p.), rats received intratracheal injection of $300 \mu \mathrm{L}$ of normal saline (control group), $300 \mu \mathrm{L}\left(3 \times 10^{9}\right.$ plaque-forming units $\left.(\mathrm{PFU})\right)$ of Ad-EGFP (Ad-EGFP group), or $300 \mu \mathrm{L}\left(3 \times 10^{9} \mathrm{PFU}\right)$ of Ad-siTLR4 (Ad-siTLR4 group). Seven days later, animals in the Ad-EGFP and Ad-siTLR4 group received intravenous injection of LPS $(5 \mathrm{mg} / \mathrm{kg})$. In the control group, normal saline $(5 \mathrm{mg} / \mathrm{kg})$ was injected intravenously and the lungs were collected $4 \mathrm{~h}$ later by thoracotomy. The right lung was fixed in $4 \%$ formaldehyde before routine processing and paraffin embedding.

2.5. Total Protein Content in Bronchoalveolar Lavage Fluid. Bronchoalveolar lavage fluid (BALF) was obtained by cannulating the trachea with a needle and by infusing the lungs five times with $1 \mathrm{~mL}$ of saline. Approximately $4.0 \mathrm{~mL}$ of BALF was obtained from each rat and homogenized on ice in $3 \mathrm{~mL}$ of cold $\left(4^{\circ} \mathrm{C}\right)$ normal saline $(0.9 \% \mathrm{NaCl})$ three times. The homogenate was centrifuged at $1000 \times \mathrm{g}$ for $10 \mathrm{~min}$ at $4^{\circ} \mathrm{C}$. The total protein concentration of BALF was determined by using the Bradford assay (Bio-Rad, Hercules, Calif).

\subsection{Analysis of Lung Injury in ALI Rat}

2.6.1. Wet-to-Dry Ratio of the Lung. Pulmonary edema was estimated by wet-to-dry ratio of the lung, a technique commonly used for the assessment of experimental lung injury [2]. Lungs were removed and rinsed briefly in PBS. The water on the lung was removed and then weighed as the "wet" weight. Lungs were then dried in an oven at $200^{\circ} \mathrm{C}$ for 2 days and weighed again as the "dry" weight. Wet-to-dry ratio was calculated as "wet" weight/"dry" weight.

2.6.2. Histological Examination of the Lung. The lungs were fixed in $4 \%$ paraformaldehyde with $1.5 \%$ picric acid in $0.16 \mathrm{M}$ phosphate buffer ( $\mathrm{pH} 7.2 \sim 7.4)$ overnight at $4^{\circ} \mathrm{C}$, embedded in paraffin, and cut into $5-\mu \mathrm{m}$ sections followed 
by hematoxylin and eosin (HE) staining for histological examination.

2.6.3. Myeloperoxidase (MPO) Assay. MPO activity was measured as described previously [21, 23]. Upon thawing, the lung tissues were homogenized in PBS $(20 \mathrm{mM}, \mathrm{pH} 7.4)$ and centrifuged at $30000 \times \mathrm{g}$ for $30 \mathrm{~min}$. The pellet was resuspended in fresh PBS (50 mM, pH 6.0) containing 0.5\% hexadecyltrimethyl ammonium bromide. The MPO activity of cell suspension was assayed by measuring the absorbance spectrophotometrically at $460 \mathrm{~nm}$, using $0.167 \mathrm{mg} / \mathrm{mL} \mathrm{O-}$ dianisidine hydrochloride and $0.0005 \%$ hydrogen peroxide. One unit of MPO activity was defined as the quantity of enzyme degrading $1 \mu \mathrm{mol}$ of peroxide per min, and data were expressed as units per gram of wet tissue.

2.7. Real-Time PCR. Real-time PCR was performed to detect the mRNA expression of TLR4 in the lung tissues. Total RNA was extracted from the lung using TRIzol reagent (Invitrogen, Carlsbad, CA). The isolated RNA was treated with DNase I at $37^{\circ} \mathrm{C}$ for $30 \mathrm{~min}$ before reverse transcription was performed using a high capacity cDNA archive kit (TaKaRa, Japan). The PCR primers were as follows: $5^{\prime}$-CGG GAG CTC TGA ATG CTC TCT TGC ATC TGG CTG GC-3' (forward) and $5^{\prime}$-CGG GTC GAC GCC ATA CAA TTC GAC CTG CTG-3' (reverse). The real-time PCR was performed using the MasterMix System (Roche, Switzerland).

2.8. Western Blot Assay. The protein expression of TLR4 in the AMs after transfection was determined by western blot assay using Nupage $4 \sim 12 \%$ Bis-Tris (Invitrogen, Carlsbad, CA) as previously described [24]. Anti-TLR4 antibody was purchased from Santa Cruz Biotechnology (Santa Cruz, USA).

2.9. Immunohistochemistry for TLR4 in the Lung. The lungs were fixed in $4 \%$ paraformaldehyde and $1.5 \%$ picric acid in $0.16 \mathrm{M}$ PBS ( $\mathrm{pH} 7.2 \sim 7.4$ ) overnight at $4^{\circ} \mathrm{C}$. Samples were removed and postfixed in the same fixative for $3 \mathrm{~h}$ and then in 15\% sucrose overnight. Transverse lung sections $(30 \mu \mathrm{m})$ were obtained with a cryostat and processed for immunofluorescence staining. All of sections were blocked with $2 \%$ goat serum in $0.3 \%$ Triton X-100 for $1 \mathrm{~h}$ at room temperature and incubated with anti-TLR4 antibody (anti-rabbit, 1:400; Santa Cruz, USA) over two nights at $4^{\circ} \mathrm{C}$. The sections were then incubated with Cy3-conjugated secondary antibody $(1: 300$; Santa Cruz, USA) for $1 \mathrm{~h}$ at room temperature and with a mixture of FITC- and Cy3conjugated secondary antibodies for $1 \mathrm{~h}$ at room temperature. Subsequently, these sections were observed under an Olympus fluorescence microscope (Olympus, Japan), and representative images were captured.

2.10. Enzyme-Linked Immunosorbant Assay (ELISA) for Cytokine Detection. To detect the IL- $1 \beta$ and TNF- $\alpha$ protein levels, the cell supernatant and BALF of rats were analyzed by using the ELISA kits specific for these cytokines (Peprotech,
UK). The ELISA of BAL fluid was performed according to the manufacturer's instructions.

\section{Results}

3.1. LPS-Induced Increase of TLR4 Expression Was Reduced by Ad-siTLR4 In Vitro. Before LPS induction, AMs were transfected with Ad-siTLR4 for $12 \mathrm{~h}, 24 \mathrm{~h}$, and $48 \mathrm{~h}$. Cells transfected with Ad-EGFP served as controls. Real-time PCR showed Ad-siTLR4 significantly downregulated the mRNA expression of TLR 4 at $12 \mathrm{~h}, 24 \mathrm{~h}$, and $48 \mathrm{~h}$ after transfection when compared with the Ad-EGFP group (Figure 1(b)). Western blot assay showed TLR4 protein expression was also decreased at $24 \mathrm{~h}$ and $48 \mathrm{~h}$ after transfection (Figure 1(a)). Furthermore, the TLR4 expression was decreased in a timedependent manner following Ad-siTLR4 transfection. TLR4 signal pathway after Ad-siTLR4 transfection in vitro was also evaluated via detecting the production of proinflammatory cytokines, including TNF- $\alpha$ and IL- $1 \beta$. Results revealed LPS could induce the production of these cytokines in vitro, which was associated with the upregulation of TLR4 expression (Figures 1(c) and 1(d)). Following transfection with Ad-siTLR4, AMs presented significantly lower level of cytokines when compared with the Ad-EGFP group, which was also accompanied by the down-regulation of TLR4 expression.

3.2. LPS-Induced TLR4 Expression Was Inhibited by Intratracheal Injection of Ad-siTLR4 in Rats. The silencing of TLR4 gene by Ad-siTLR4 was also investigated in rats with LPSinduced ALI. Four hours after LPS administration, the lungs were collected for real-time PCR assay, immunofluorescence staining as well as other molecular biological detections. Results showed that TLR4 mRNA expression increased in the Ad-EGFP group as compared to the control group. In the AdsiTLR4 group, the TLR4 mRNA expression was dramatically decreased when compared with Ad-EGFP group $(P<$ 0.05) (Figure 2(a)). Immunofluorescence staining showed the TLR4 protein expression in the lung of different groups was consistent with that in real-time PCR assay (Figures 2(b), 2(c), and 2(d)). In the Ad-EGFP group, EGFP was mainly found in the bronchus epithelial cells and alveolar epithelial cells after intratracheal injection (Figure 3), indicating that the recombinant adenovirus could be delivered to the target cells through intratracheal injection. The LPS-induced increase of TLR4 protein expression was reduced significantly following Ad-siTLR4 transfection. Exposure to AdEGFP had no effect on the TLR4 expression. These findings imply intratracheal injection of Ad-siTLR4 can interfere with the TLR4 mRNA transcription, and then inhibit its protein expression.

\subsection{TLR4-Related Proinflammatory Cytokines Were Decreased} after Knockdown of TLR4 Gene. Inflammatory cytokines, such as TNF- $\alpha$ and IL-1, were shown to play key roles in the pathophysiology of LPS-induced ALI. We measured the levels of these cytokines in the BALF after LPS treatment. When compared with the control group, rats receiving LPS 


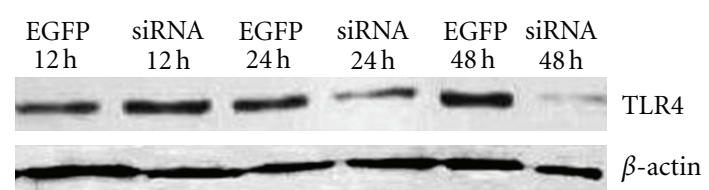

(a)

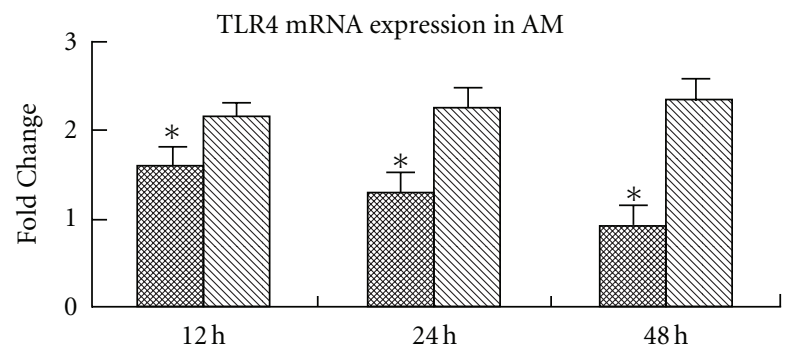

(b)

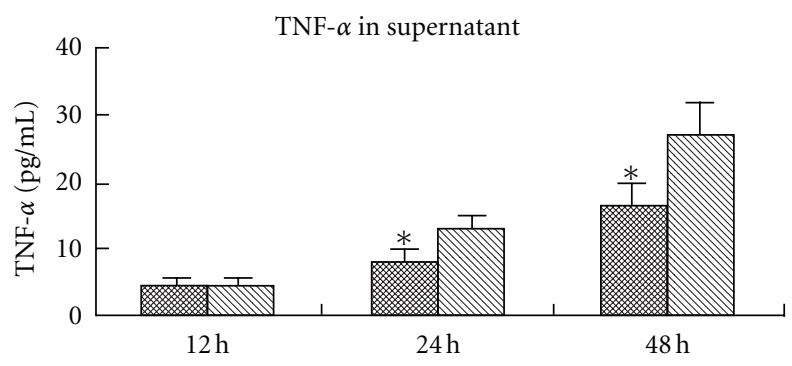

(c)

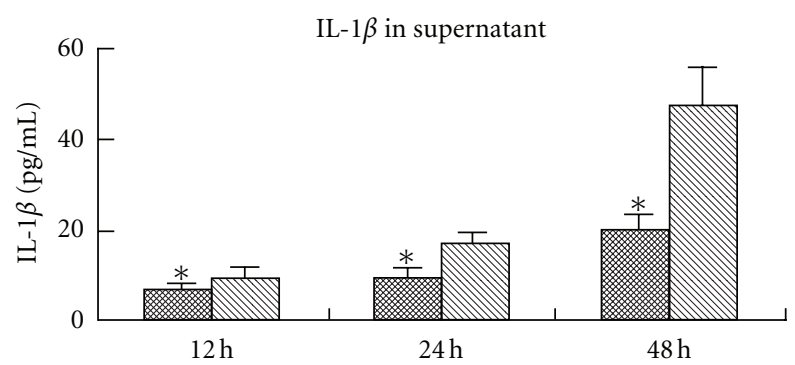

Ad-siTLR4

Ad-EGFP

(d)

FIgure 1: (a) Western blot assay of TLR4 following Ad-siTLR4 or Ad-EGFP pretreatment in LPS treated AMs. (b) Real-time PCR analysis of TLR4 mRNA expression in LPS-treated AMs. (c) and (d) levels of TNF- $\alpha$ and IL- $1 \beta$ in the supernatant. ${ }^{*} P<0.05$ versus AdEGFP group.

treatment in the Ad-EGFP group had markedly increased expressions of TNF- $\alpha$ and IL-1. In Ad-siTLR 4 group, TNF$\alpha$ and IL- 1 expressions markedly decreased at $4 \mathrm{~h}$ after LPS treatment as compared to the Ad-EGFP group (Figure 4).

3.4. Ad-siTLR4 Targeting TLR4 Attenuated LPS-Induced ALI. In the present study, pathological features of the lungs of rats with LPS-induced lung injury were observed following Ad-siTLR4 treatment. Lung histological examination showed that, in animals treated with saline, the lung was almost

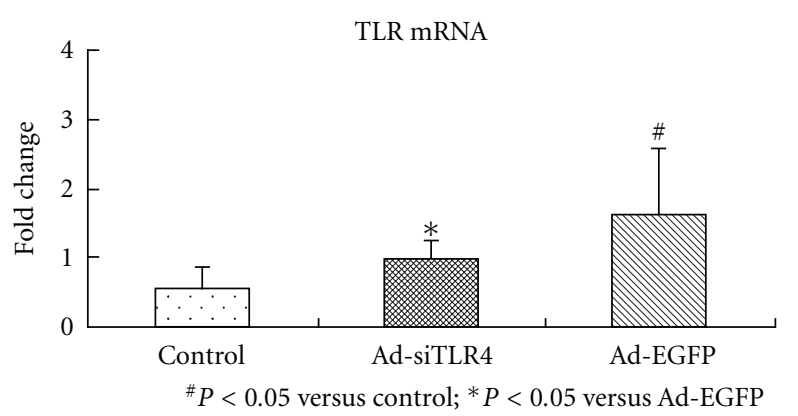

(a)

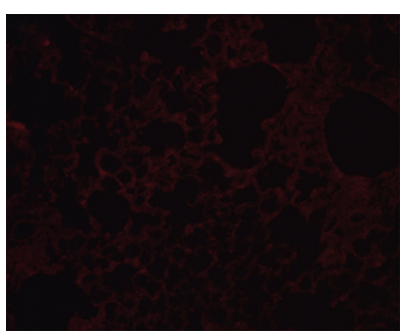

(b)

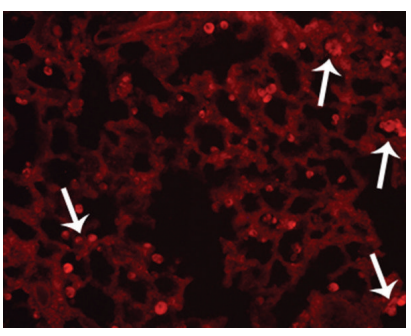

(c)

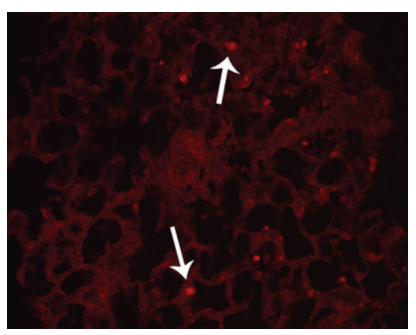

(d)

Figure 2: (a) mRNA level of TLR4 in the lung in control group, Ad-EGFP group, Ad-siTLR4 group following LPS treatment. (b), (c), and (d) represent TLR4 protein expression in the lung tissues in control group, Ad-EGFP group, and Ad-siTLR4 group, respectively. ${ }^{*} P<0.05$ versus Ad-EGFP group, ${ }^{*} P<0.05$ versus control group. White arrow shows the positive cells.

intact (Figure 5(a)). By contrast, in the Ad-EGFP group, LPS treatment significantly increased the exudation and thickened the alveolar walls accompanied by alveolus congestion, hemorrhage as well as massive neutrophil infiltration (Figure 5(b)). The pathological changes in the lungs were not obvious in the Ad-siTLR4 group (Figure 5(c)). The wet/dry ratio of the lungs was measured to represent the extent of lung edema. Results revealed LPS dramatically increased the wet/dry ratio, while Ad-siTLR4 treatment markedly reduced the lung edema when compared with the Ad-EGFP group (Figure 5(d)). Moreover, in the Ad-EGFP group, the content of total proteins was significantly increased as compared to the control group. However, treatment with Ad-siTLR4 markedly decreased the total protein content in the BALF, indicating intratracheal injection of Ad-siTLR4 can improve the LPS-induced microvascular leakage (Figure 5(e)). In order to examine the leukocyte infiltration in response to LPS, the lung MPO activity, an index of neutrophil sequestration, was determined. Findings displayed there was 


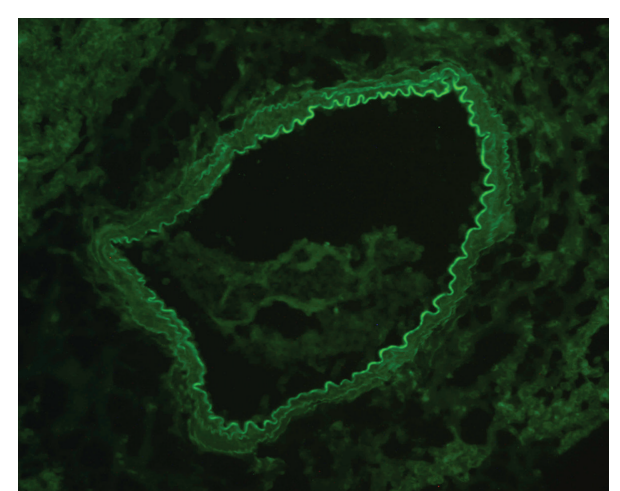

(a)

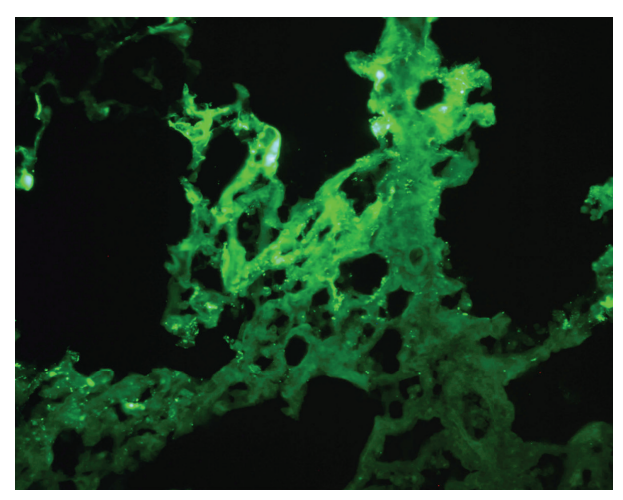

(b)

FIGURE 3: (a) and (b) represent adenovirus transfected into bronchus epithelial cells (a) and alveolar epithelial cells (b), respectively. The green fluorescence represents the adenovirus.

no significant difference in the lung MPO activity between the saline group and the Ad-siTLR4 group indicating no obvious neutrophil infiltration into both the alveolar space and the whole lung, while MPO activity in the Ad-EGFP group was significantly increased when compared with the former two groups (Figure 5(f)). These observations indicate that Ad-siTLR4 treatment can improve the lung injury in response to LPS through reducing microvascular damage, decreasing neutrophil influx, and improving lung histology.

\section{Discussion}

Results in the present study revealed Ad-siTLR4 could interfere with the TLR4 expression in vivo and in vitro, and treatment with Ad-siTLR4 could improve the LPS-induced ALI. To our knowledge, this study is for the first time to report siRNA targeting TLR4 as a therapeutic strategy for rat ALI. Our findings showed Ad-siTLR4 targeting TLR4 could directly reduce the TLR4 expression after LPS treatment both in vivo and in vitro. Moreover, this treatment also attenuated pulmonary injury by decreasing the production of proinflammatory cytokines in the lungs and alleviating the pathological inflammation. The main findings of this study suggest TLR4 plays a critical role in the LPS-induced ALI and gene therapy targeting TLR4 may become a promising strategy in the prevention of LPS-induced inflammatory response.

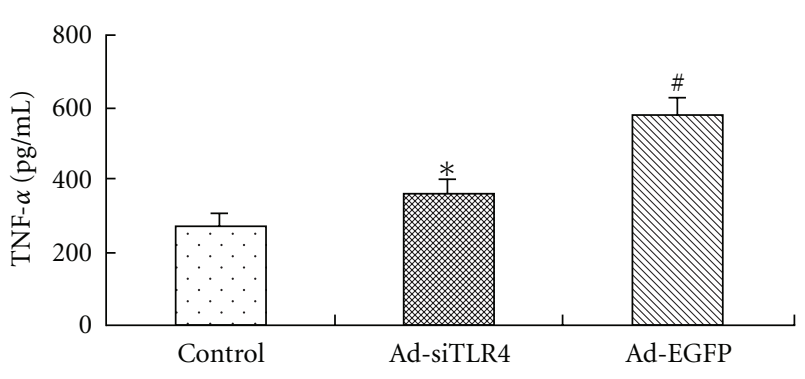

(a)

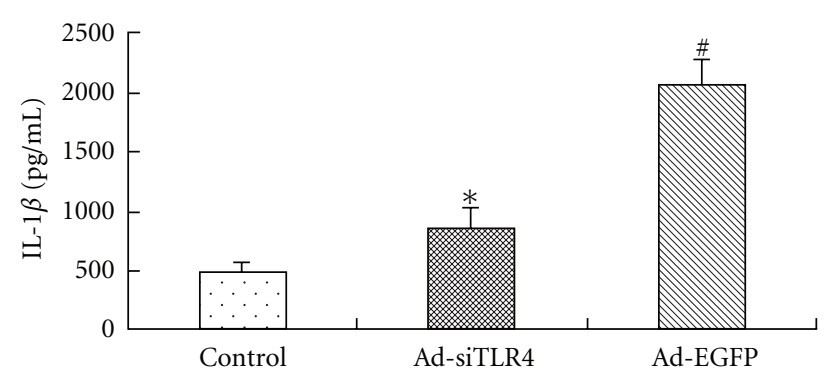

(b)

FIGURE 4: Effect of Ad-siTLR4 transfection on the expression of proinflammatory cytokines in the lung of ALI rats. The TNF$\alpha$ (a) and IL- $1 \beta$ (b) were determined by ELISA at $4 \mathrm{~h}$ after LPS administration. Data were expressed as fold change when compared with saline-treated animals. ${ }^{*} P<0.05$ versus Ad-EGFP group, ${ }^{\#} P<0.05$ versus control group.

ALI or ARDS is one of clinical conditions associated with sepsis in humans and characterized by the microvascular protein leakage, neutrophil influx, excessive production of proinflammatory mediators, and poor lung function [12, $19,25]$. Infection with gram-negative bacteria may lead to the development of sepsis syndrome as a consequence of excessive host response to LPS in humans. Increasing evidence demonstrates that TLR4 is required for the innate immune response to LPS from gram-negative bacteria. The potential mechanism of TLR4 mediating the development of ALI involves LPS signaling transduction [2628]. Binding of LPS to TLR4 in conjunction with other molecules (LBP, CD14, and MD2) is a crucial step for initiating intracellular signal transduction that accounts for its multiple biological effects leading to ALI. Upon LPS recognition, TLR4 interacts with its downstream MyD88 (myeloid differentiation primary response gene 88 ) adaptor domain $[29,30]$ and activates intracellular signal molecule including TRAF6 (TNF receptor-associated factor 6) and IKK $\alpha, \beta$ (I $\kappa \mathrm{B}$ kinase) to form a complex and phosphorylate $\mathrm{I} \kappa \mathrm{B}[31,32]$. This phosphorylation leads to the degradation of $\mathrm{I} \kappa \mathrm{B}$ with subsequent translocation of NF- $\kappa \mathrm{B}$, which controls the expression of proinflammatory cytokines including IL- $1 \beta$, and TNF- $\alpha$ [33]. Based on well-known LPS/TLR4 signal transduction pathway, the proinflammatory cytokines TNF- $\alpha$ and IL- $1 \beta$ were employed to assess the cytokine response following LPS exposure. Our results also demonstrated systemic administration of LPS led to the significant increase of TLR4 expression as well as the production of 


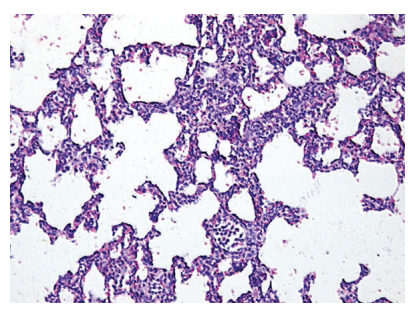

(a)

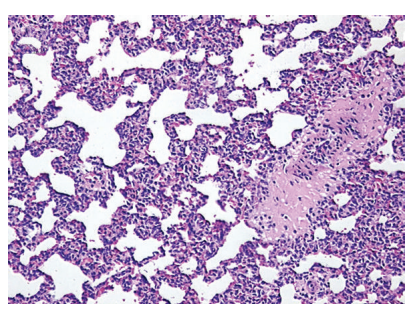

(b)

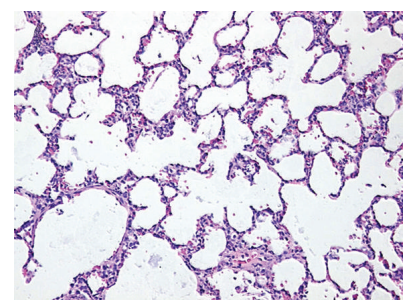

(c)

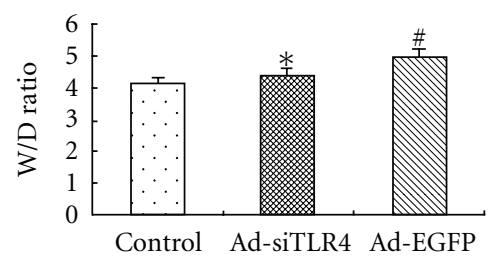

(d)

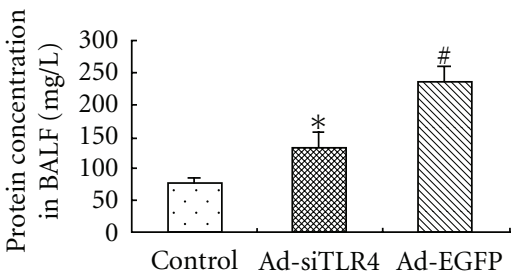

(e)

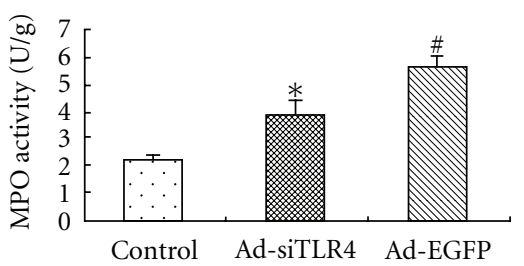

(f)

FIGURE 5: Effects of Ad-siTLR4 on LPS-induced ALI. Lungs were collected at $4 \mathrm{~h}$ after injection of normal saline or LPS for histological examination $(a, b, c)$. Sections were stained with hematoxylin-eosin. Magnification: $\times 100 \mathrm{Wet} /$ Dry ratio $(\mathrm{d})$, total protein content in BALF (e) as well as MPO activity (f) were measured to determine the lung edema, microvascular permeability, and neutrophil sequestration, respectively. ${ }^{*} P<0.05$ versus Ad-EGFP group, ${ }^{\#} P<0.05$ versus control group.

IL- $1 \beta$ and TNF- $\alpha$, which were markedly reduced following pretreatment with Ad-siTLR4 targeting TLR4 in vitro and in vivo. In order to confirm that this inflammatory response was provoked by LPS instead of Ad-siTLR4 itself, animals were also injected with Ad-siTLR4 as controls. When compared with LPS administration alone, Ad-siTLR4 injection alone could not evoke the abovementioned immune inflammatory response, indicating that LPS evoked these inflammatory pathological changes.

The role of TLR4 in the development of ALI is still controversial. A study demonstrated that factors other than TLR4 were involved in the secretion of TNF- $\alpha$ and MIP-2 in the lungs in response to inhaled LPS and the authors postulated that this response was due to low levels of contamination by the E. coli LPS [11]. In addition, another study reported that TLR4 played a role in lung inflammation induced by hemorrhage following LPS injection, but the authors neither examined the cytokine profiles (such as TNF- $\alpha$ ) nor determined the lung histopathology [34]. This possibility is unlikely since Jeyaseelan et al. [12] showed that TLR4 is solely responsible for the induction of ALI via activation of NF- $\kappa \mathrm{B}$ with subsequent production of cytokines. In present study, Ad-siTLR4 was prepared for the treatment of LPS-induced ALI. Our results confirmed that upregulation of TNF- $\alpha$ and IL- $1 \beta$ was dependent on TLR4 overexpression as a response to LPS. Thereby, TLR4 may be a valuable target in the immunotherapy of acute lung inflammation due to endotoxins. Our results also indicated markedly mild inflammatory response including significantly low levels of TNF- $\alpha$ and IL-1 $\beta$ in the lung, and low total protein level in BALF, decreased MPO activity, as well as reduced wet/dry ratio following Ad-siTLR4 treatment when compared with LPS group. Furthermore, when compared with control group, no significant changes were found in the lung histopathology in the Ad-siTLR4 group. These findings imply TLR4 modulation in ALI in response to LPS may have therapeutic value.

In the application of siRNA, the specificity, efficiency, and stability of siRNA in target cells should be considered [35]. Therefore, to successfully achieve gene silencing, carefully designed siRNA and effective delivery to the target lung cells are paramount. In the present study, 21 duplexes with a 19-base central double-stranded region and terminal 2base $3^{\prime}$ overhangs were applied. This siRNA mimics the naturally occurring molecules produced by dicer processing in vivo and can prevent off-target effects. It has been reported that siRNA mediated down-regulation of gene expression is transient and can last for only $3 \sim 5$ days in vitro. Our results demonstrated that the adenovirus carrying siRNA targeting TLR4 could exert therapeutic effect for at least 7 days in vivo and the gene silencing lasts for at least $48 \mathrm{~h}$ in vitro. To obtain high performance of interference, adenovirus expressing siRNA was delivered to the lungs via intratracheal instillation to directly colonize in target cells of lung, with prolonged therapeutic efficacy. The presence of EGFP showed that adenovirus-vector system was delivered to the bronchoepithelial cells, pulmonary alveoli epithelial cells as well as macrophages at 7 days after instillation indicating that the vector was integrated into the target cells with relatively long expression. In vitro, downregulation of TLR4 expression by Ad-siTLR4 was shown in the pulmonary macrophages following LPS treatment. Western blot assay of collected cells also exhibited decreased TLR4 expression in a time-dependent manner. Moreover, we showed a marked reduction of TLR4 expression following 
Ad-siTLR4 accompanied by compromised TLR4 functions which were demonstrated by the reduced production of TNF- $\alpha$ and IL- $1 \beta$ following LPS stimulation in vitro. These results demonstrated that the siRNA targeting TLR4 gene was expressed by the adenovirus knock-down TLR4 gene efficiently.

In summary, our results demonstrate that TLR4 mediates the LPS induced ALI in a rat model. Furthermore, delivering siRNA targeting TLR4 to target cells in the lung exhibits therapeutic effect on ALI. Collectively, the findings in the present study suggest TLR4 is an attractive target for immunomodulation and support that inhibiting TLR4 expression by using siRNA is a promising therapeutic strategy to minimize ALI.

\section{Abbreviations}

ALI: Acute lung injury

TLR4: Toll-like receptor 4

ARDS: Acute respiratory distress syndrome

LPS: Lipopolysaccharide

siRNA: Small interfering RNAs

RNAi: RNA interference

CNV: Choroidal neovascularization

EGFP: Enhanced green fluorescent protein

WT: Wide type

MOI: Multiplicity of infection

BALF: Bronchoalveolar lavage fluid

HE: Hematoxylin and eosin.

\section{Conflict of Interests}

The authors declare no conflict of interests.

\section{Acknowledgment}

This work was supported by National Natural Science Foundation of China.

\section{References}

[1] A. Pontes-Arruda, S. DeMichele, A. Seth, and P. Singer, "The use of an inflammation-modulating diet in patients with acute lung injury or acute respiratory distress syndrome: a metaanalysis of outcome data," Journal of Parenteral and Enteral Nutrition, vol. 32, no. 6, pp. 596-605, 2008.

[2] J. S. Wu, L. Sheng, S. H. Wang et al., "The impact of clinical risk factors in the conversion from acute lung injury to acute respiratory distress syndrome in severe multiple trauma patients," Journal of International Medical Research, vol. 36, no. 3, pp. 579-586, 2008.

[3] R. C. Gillis, L. J. Weireter Jr., R. C. Britt, F. J. Cole Jr., J. N. Collins, and L. D. Britt, "Lung protective ventilation strategies: have we applied them in trauma patients at risk for acute lung injury and acute respiratory distress syndrome?" American Surgeon, vol. 73, no. 4, pp. 347-350, 2007.

[4] M. Karabeyoglu, B. Kocer, U. Ozel et al., "The protective effect of ethyl pyruvate on lung injury after burn in rats," Saudi Medical Journal, vol. 28, no. 10, pp. 1489-1492, 2007.
[5] K. L. Brigham and B. Meyrick, "Endotoxin and lung injury," American Review of Respiratory Disease, vol. 133, no. 5, pp. 913-927, 1986.

[6] F. Chabot, J. A. Mitchell, J. M. C. Gutteridge, and T. W. Evans, "Reactive oxygen species in acute lung injury," European Respiratory Journal, vol. 11, no. 3, pp. 745-757, 1998.

[7] K. Kubo, T. Amari, T. Kaneki et al., "A 21-aminosteroid, U74006F, attenuates endotoxin-induced lung injury in awake sheep," Respirology, vol. 4, no. 2, pp. 167-172, 1999.

[8] R. Rabinovici, P. J. Bugelski, K. M. Esser, L. M. Hillegass, J. Vernick, and G. Feuerstein, "ARDS-like lung injury produced by endotoxin in platelet-activating factor-primed rats," Journal of Applied Physiology, vol. 74, no. 4, pp. 1791-1802, 1993.

[9] K. Hoshino, O. Takeuchi, T. Kawai et al., "Cutting edge: tolllike receptor 4 (Tlr4)-deficient mice are hyporesponsive to lipopolysaccharide evidence for Tlr4 as the Lps gene product," Journal of Immunology, vol. 162, no. 7, pp. 3749-3752, 1999.

[10] A. Poltorak, X. He, I. Smirnova et al., "Defective LPS signaling in C3H/HeJ and C57BL/10ScCr mice: mutations in Tlr4 gene," Science, vol. 282, no. 5396, pp. 2085-2088, 1998.

[11] E. Lorenz, M. Jones, C. Wohlford-Lenane et al., "Genes other than Tlr4 are involved in the response to inhaled LPS," American Journal of Physiology, vol. 281, no. 5, pp. L1106L1114, 2001.

[12] S. Jeyaseelan, H. W. Chu, S. K. Young, M. W. Freeman, and G. S. Worthen, "Distinct roles of pattern recognition receptors CD14 and Toll-like receptor 4 in acute lung injury," Infection and Immunity, vol. 73, no. 3, pp. 1754-1763, 2005.

[13] M. Schlee, V. Hornung, and G. Hartmann, "siRNA and isRNA: two edges of one sword," Molecular Therapy, vol. 14, no. 4, pp. 463-470, 2006.

[14] J. Moffat and D. M. Sabatini, "Building mammalian signalling pathways with RNAi screens," Nature Reviews Molecular Cell Biology, vol. 7, no. 3, pp. 177-187, 2006.

[15] K. Okamura, W. J. Chung, and E. C. Lai, "The long and short of inverted repeat genes in animals: microRNAs, mirtrons and hairpin RNAs," Cell Cycle, vol. 7, no. 18, pp. 2840-2845, 2008.

[16] K. Okamura and E. C. Lai, "Endogenous small interfering RNAs in animals," Nature Reviews Molecular Cell Biology, vol. 9, no. 9, pp. 673-678, 2008.

[17] S. J. Reich, J. Fosnot, A. Kuroki et al., "Small interfering RNA (siRNA) targeting VEGF effectively inhibits ocular neovascularization in mouse model," Molecular Vision, vol. 9, pp. 210-216, 2003.

[18] D. Jiang, J. Liang, Y. Li, and P. W. Noble, "The role of Toll-like receptors in non-infectious lung injury," Cell Research, vol. 16, no. 8, pp. 693-701, 2006.

[19] Y. Imai, K. Kuba, G. G. Neely et al., "Identification of oxidative stress and Toll-like receptor 4 signaling as a key pathway of acute lung injury," Cell, vol. 133, no. 2, pp. 235-249, 2008.

[20] F. X. Wu, J. J. Bian, X. R. Miao et al., "Intrathecal siRNA against toll-like receptor 4 reduces nociception in a rat model of neuropathic pain," International Journal of Medical Sciences, vol. 7, no. 5, pp. 251-259, 2010.

[21] S. E. Kim, T. T. T. Thuy, J. H. Lee et al., "Simvastatin inhibits induction of matrix metalloproteinase-9 in rat alveolar macrophages exposed to cigarette smoke extract," Experimental and Molecular Medicine, vol. 41, no. 4, pp. 277287, 2009.

[22] J. A. Nick, S. K. Young, P. G. Arndt et al., "Selective suppression of neutrophil accumulation in ongoing pulmonary inflammation by systemic inhibition of p38 mitogen-activated protein kinase," Journal of Immunology, vol. 169, no. 9, pp. 5260-5269, 2002. 
[23] G. Andonegul, S. M. Goyert, and P. Kubes, "Lipopolysaccharide-induced leukocyte-endothelial cell interactions: a role for CD14 versus toll-like receptor 4 within microvessels," Journal of Immunology, vol. 169, no. 4, pp. 2111-2119, 2002.

[24] V. V. Sumbayev, "LPS-induced Toll-like receptor 4 signalling triggers cross-talk of apoptosis signal-regulating kinase 1 (ASK1) and HIF-1 $\alpha$ protein," FEBS Letters, vol. 582, no. 2, pp. 319-326, 2008.

[25] T. R. Martin and M. M. Wurfel, "A TRIFfic perspective on acute lung injury," Cell, vol. 133, no. 2, pp. 208-210, 2008.

[26] A. Bhattacharyya, S. K. Pathak, A. Banerjee, S. Basu, and M. Kundu, "Tlr4-dependent NF- $\kappa$ B activation and mitogen- and stress-activated protein kinase 1-triggered phosphorylation events are central to Helicobacter pylori peptidyl prolyl cis-, trans-isomerase (HP0175)-mediated induction of IL-6 release from macrophages," Journal of Immunology, vol. 177, no. 11, pp. 7950-7958, 2006.

[27] M. A. Chase, D. S. Wheeler, K. M. Lierl, V. S. Hughes, H. R. Wong, and K. Page, "Hsp72 induces inflammation and regulates cytokine production in airway epithelium through a Tlr4and NF- $\kappa$ B-dependent mechanism," Journal of Immunology, vol. 179, no. 9, pp. 6318-6324, 2007.

[28] M. Yu, D. Shao, J. Yang, S. Feng, and J. Xu, "Ketamine suppresses intestinal Tlr4 expression and NF- $\kappa \mathrm{B}$ activity in lipopolysaccharide-treated rats," Croatian Medical Journal, vol. 47, no. 6, pp. 825-831, 2006.

[29] C. W. Wieland, S. Florquin, N. A. Maris et al., "The MyD88dependent, but not the MyD88-independent, pathway of Tlr4 signaling is important in clearing nontypeable Haemophilus influenzae from the mouse lung," Journal of Immunology, vol. 175, no. 9, pp. 6042-6049, 2005.

[30] Y. S. Lee, J. S. Park, J. H. Kim et al., "Smad6-specific recruitment of Smurf E3 ligases mediates TGF- $\beta 1$-induced degradation of MyD88 in Tlr4 signalling," Nature Communications, vol. 2, no. 1, pp. 460-469, 2011.

[31] O. Takeuchi and S. Akira, "Toll-like receptors; their physiological role and signal transduction system," International Immunopharmacology, vol. 1, no. 4, pp. 625-635, 2001.

[32] K. Hoshino, I. Sasaki, T. Sugiyama et al., "Critical role of I $\kappa \mathrm{B}$ kinaseain TLR7/9-induced type I IFN production by conventional dendritic cells," Journal of Immunology, vol. 184, no. 7, pp. 3341-3345, 2010.

[33] C. Gille, B. Spring, W. Bernhard et al., "Differential effect of surfactant and its saturated phosphatidylcholines on human blood macrophages," Journal of Lipid Research, vol. 48, no. 2, pp. 307-317, 2007.

[34] J. Fan, Y. Li, Y. Vodovotz, T. R. Billiar, and M. A. Wilson, "Hemorrhagic shock-activated neutrophils augment Tlr4 signaling-induced TLR2 upregulation in alveolar macrophages: role in hemorrhage-primed lung inflammation," American Journal of Physiology, vol. 290, no. 4, pp. L738-L746, 2006.

[35] S. Gupta, R. A. Schoer, J. E. Egan, G. J. Hannon, and V. Mittal, "Inducible, reversible, and stable RNA interference in mammalian cells," Proceedings of the National Academy of Sciences of the United States of America, vol. 101, no. 7, pp. 1927-1932, 2004. 


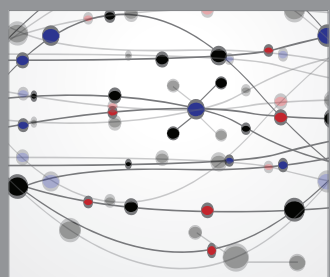

The Scientific World Journal
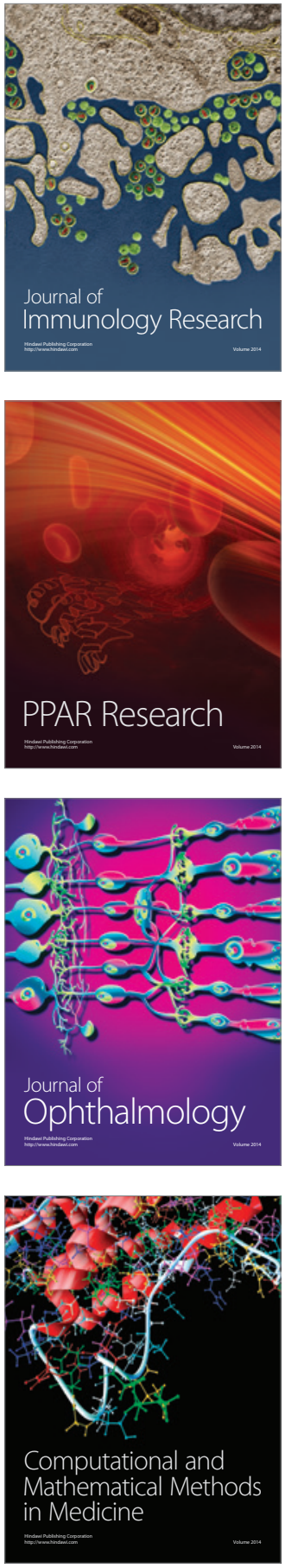

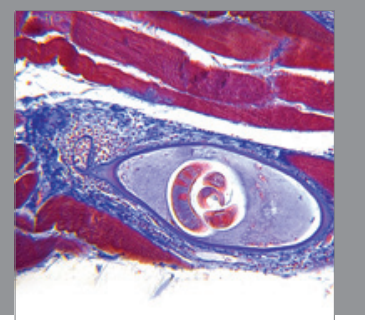

Gastroenterology

Research and Practice
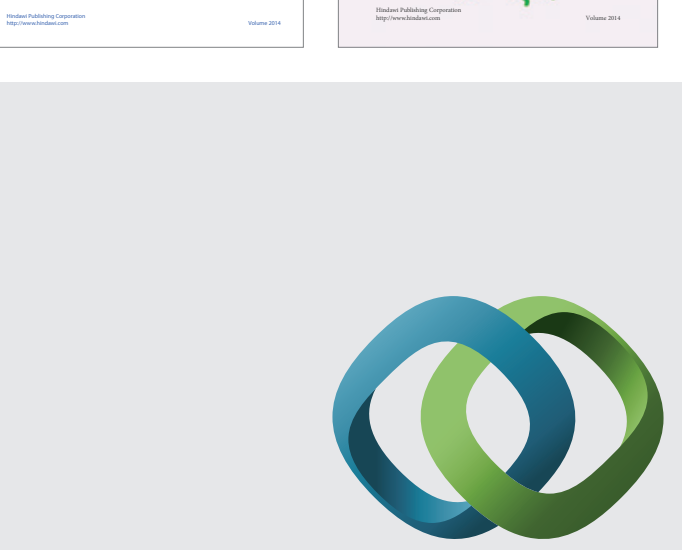

\section{Hindawi}

Submit your manuscripts at

http://www.hindawi.com
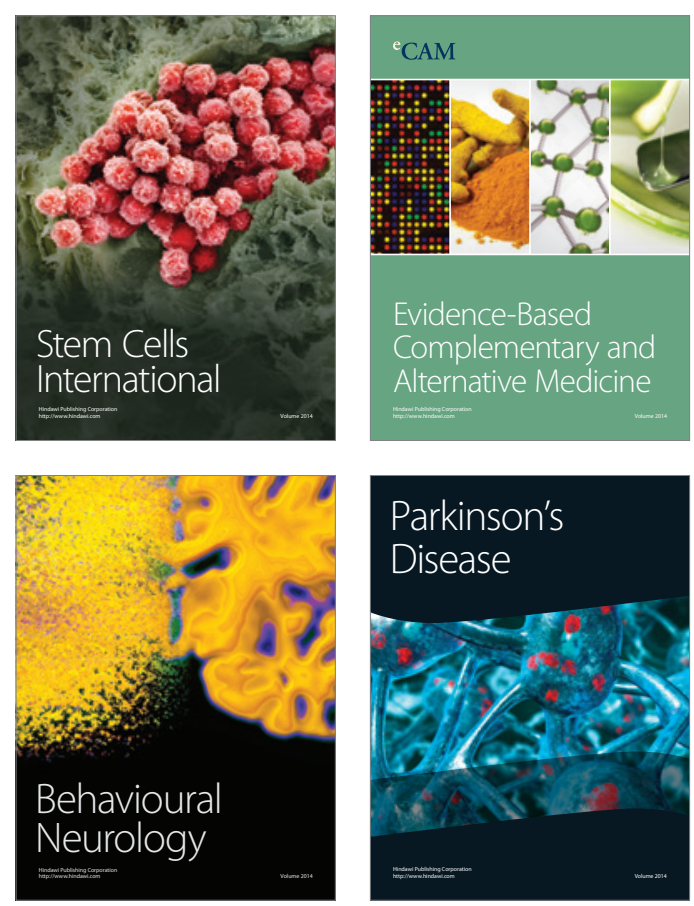

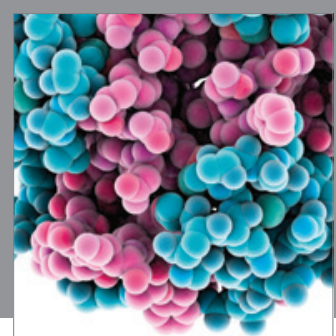

Journal of
Diabetes Research

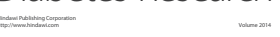

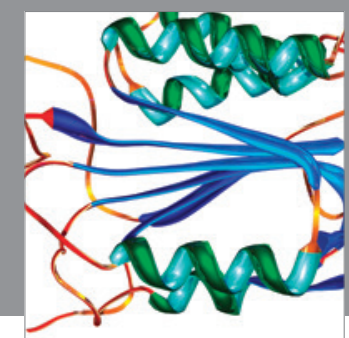

Disease Markers
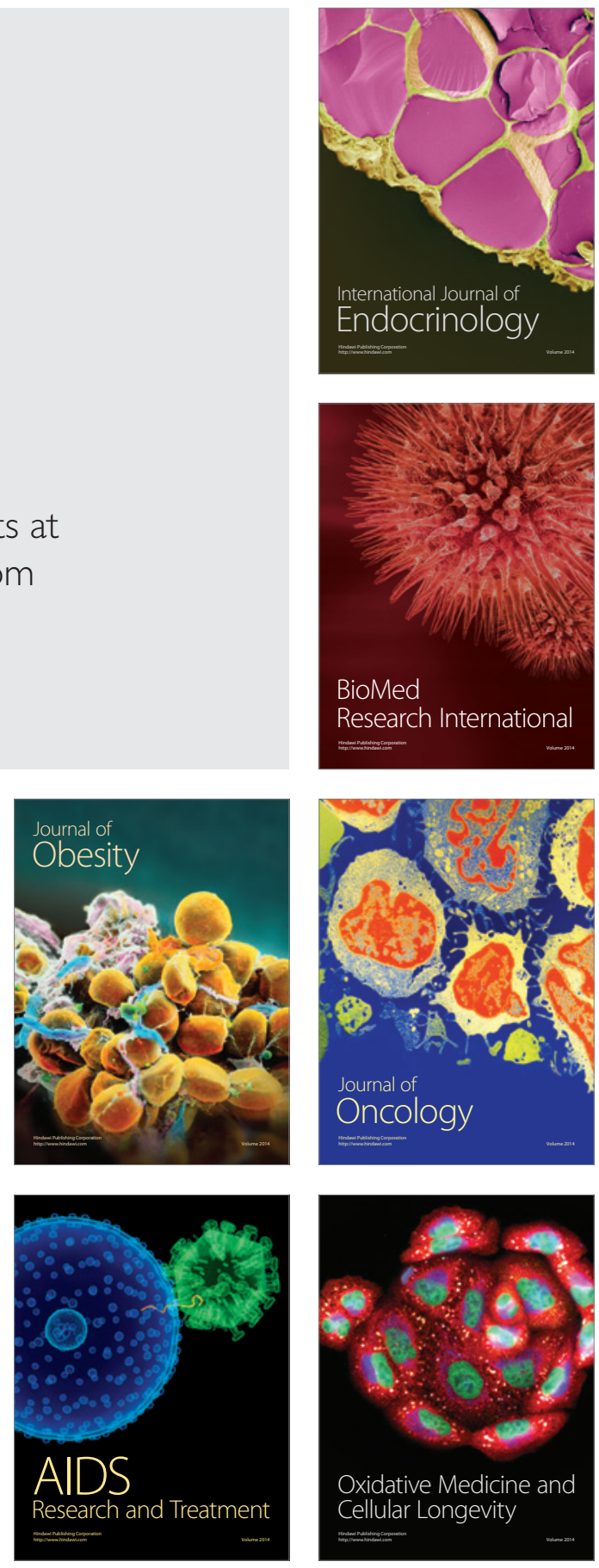